\title{
The Simulation and Research of Impedance-Matching Balance Transformer Based on Ansoft
}

\author{
SUN Pan ${ }^{1, a^{*}}$, ZHAO Jing Hong ${ }^{1, b}$ and WU Xu Sheng ${ }^{1, c}$ \\ ${ }^{1}$ College of Electrical Engineering, Naval University of Engineering, Wuhan, China, 430033 \\ a489451440@qq.com ,b2679228862@qq.com ,'Wuxusheng@163.com
}

Keywords: ANSOFT, Balance transformer, Finite-element analysis, Impedance-matching Abstract. The basic principle of impedance-matching balance transformer (IMBF)is introduced, the impedance matching conditions and current vector are given. Using ANSOFT finite-element simulation software for transformer modeling and simulation, the simulation results consistent with theory, shows the validity of the simulation method. The observation and analysis of the dynamic change of simulation parameters, can optimize the transformer design.

\section{Introduction}

With the advancement of modernization level, we have more kinds of single phase and two phase load, power is becoming more and more big, the resulting current and voltage imbalance power system became apparent[1]. Balance Transformer is a kind of transformer, which can transform three phase voltage into two mutually orthogonal phase voltage. It can effectively inhibit the negative sequence current and zero sequence current, improves the quality of power supply of electric power system, by adding harmonic suppression device can also have the effect of harmonic suppression, and it has a broad prospect of application. Studying the design and simulation methods and optimizing its comprehensive performance, will lay theoretical foundation for the practical application of balance transformer . In this paper, using Ansoft for IMBF's modeling[2], the basic performance is simulated. Through the analysis of the simulation, the results can help to optimize the performance of the transformer.

\section{The basic principle of IMBF}

The connection scheme of the IMBF is shown in figure 1. The primary windings adopts star connection method, the number of each phase winds is W1. The secondary windings adopts triangle connection method, the number of each phase winds is $\mathrm{W}_{2}$, and on the two ends of an $\mathrm{ab}$ winding phase in the middle, there is a denotation of arm which number is $\triangle \mathrm{W}=0.366 \mathrm{~W}_{2}$, forming a 90 degree phase between the output voltage of $U_{\alpha}$ and $U_{\beta}$. The equivalent impedance of primary side of the three-phase should be equal, namely $Z_{\mathrm{A} 1}=Z_{\mathrm{B} 1}=Z_{\mathrm{C} 1}=Z_{1}$, which should make the three-phase emf also symmetrical when the three phase voltage and current are symmetrical. In the winding equivalent impedance of the secondary side of the triangle , taking $Z_{\mathrm{ac}}=Z_{\mathrm{bc}}=Z_{2}$ and $Z_{\mathrm{bc}}=2.732 Z_{2}$, the winding equivalent leakage impedance of the two arms $Z_{\mathrm{da}}=Z_{\mathrm{be}}=\sigma Z_{2}$. The mutual inductance between arm winding is $X_{\mathrm{p}}=\tau Z_{2}$, we should make $\sigma+\tau=0.4226$, and $\sigma$ approaches $\tau$ in numerical . The relationship which meets the requirements of the matching of three phase current phase is shown in figure 2 .

$$
\left[\begin{array}{l}
f_{A} \\
f_{B}^{\alpha} \\
f_{C}
\end{array}\right]=\frac{1}{2 \sqrt{3} K}\left[\begin{array}{lll}
\sqrt{3}+1 & -\sqrt{3}+1 & 2 \sqrt{3} K \\
-2 & -2 & 2 \sqrt{3} K \\
-\sqrt{3}+1 & \sqrt{3}+1 & 2 \sqrt{3} K
\end{array}\right]\left[\begin{array}{l}
\alpha \\
\alpha \\
\mathcal{\beta}_{\beta}^{\alpha}
\end{array}\right]
$$



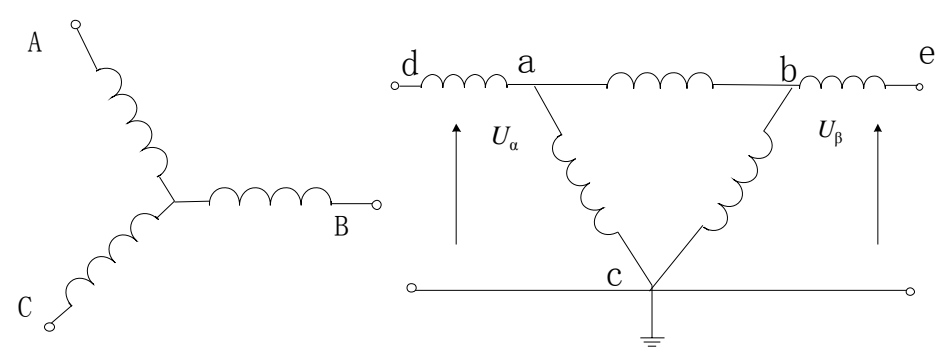

Fig. 1 Connection Scheme of IMBF windings

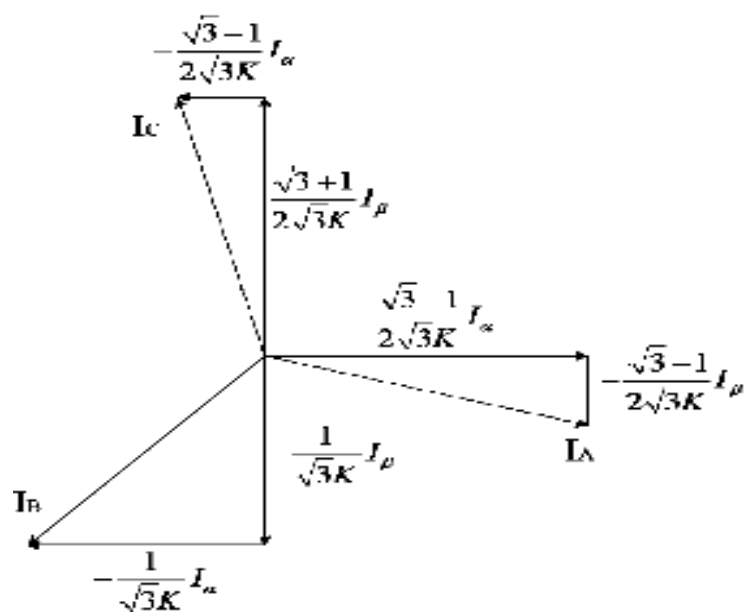

Fig. 2 Phase diagarm of the currents

\section{The transient finite-element analysis of IMBF}

Establishment of the transformer model and the setting of the solution. Creating a geometric model of the transformer. According to the power transformer design and calculation method, the parameters of the $1 \mathrm{KW}$ transformer model is calculated[3]. Creating the Maxwell 2D model, using the way of solving the whole model and specifying properties of the material, as a result ,the finite-element geometric model is shown in figure 3 .

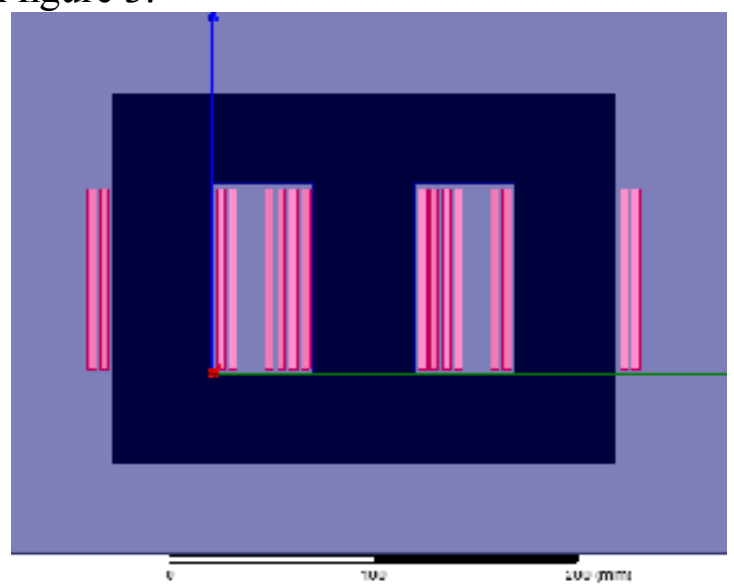

Fig. 3 The finite-element geometric model

Loading of the incentive source and the setting of the boundary conditions. Firstly, we should split phase of winding and then set up the parameters of the winding[4]. This transformer winding structure is relatively complex, so we should edit the external circuit, give the winding connection and load, and import the circuit form Edit External Circuit. Through changing the connection mode of the external circuit and the load resistance, the conditions of the transformer in the light of operation and different load can be simulated. In the calculation of finite element numerical, the thing that should 
finally be solved is the matrix equations and the boundary conditions are definite condition of the equation. Though settings dialog box by the Master Boundary, the outer region of the boundary conditions can be selected. The external circuit is shown in figure 4.

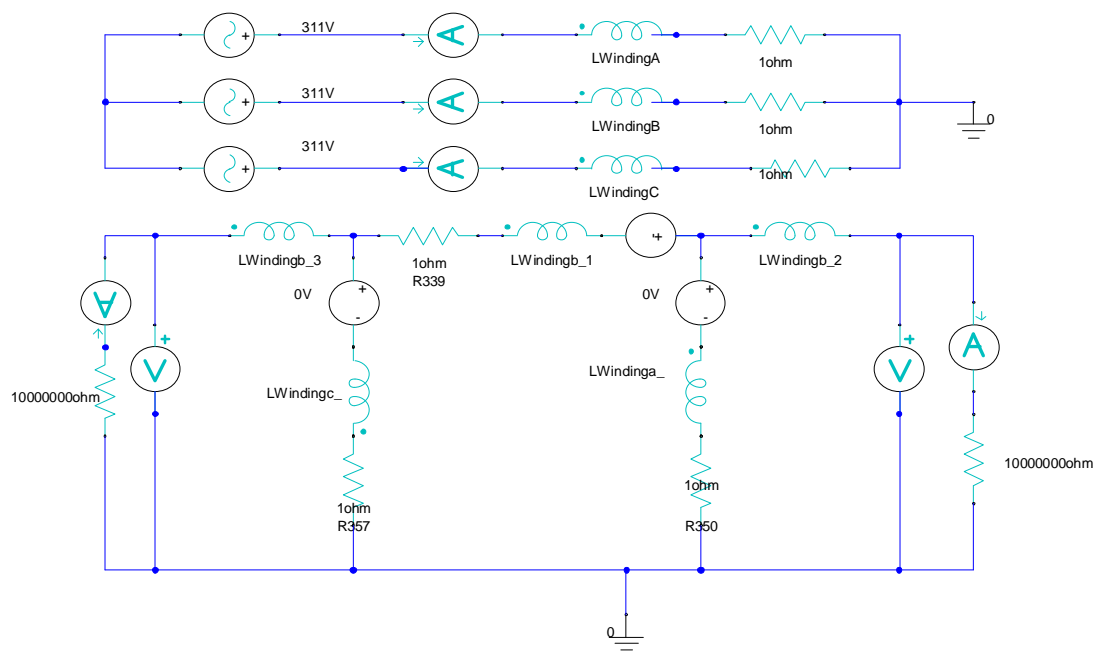

Fig. 4 The external circuit

Setting of mesh generation of transformer .Mesh generation is the most crucial step of finite element discretization ,and good mesh generation enables users to get twice the result with half the effort. Ansoft 12 mesh generation adopts the set of pyramid mesh generation, users can use the built-in adaptive cutting division directly, through On Selection, Inside Selection, Surface Approximation the three dialogs, and object the boundary, the internal and the surface subdivision rules specified object respectively. The transformer mesh map is shown in figure 5.

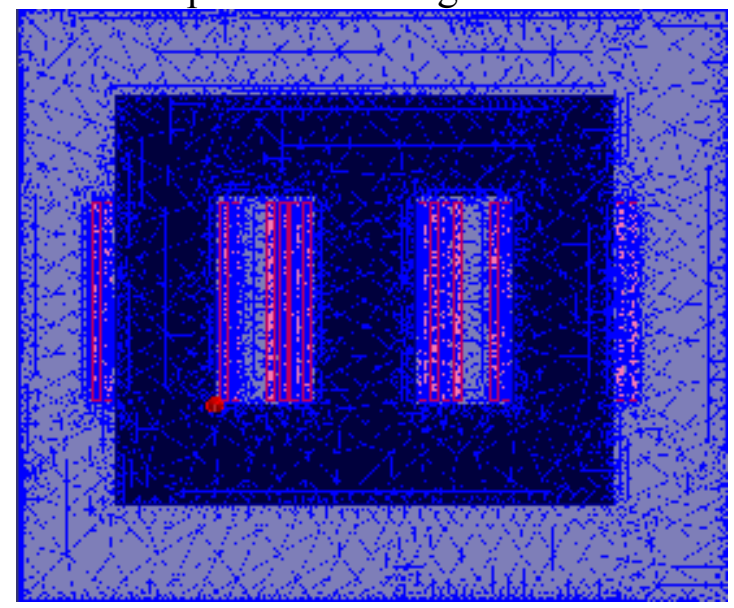

Fig. 5 The transformer mesh map

Setting of transformer solution .Perform the command of Add Solution setup and do general setting, convergence settings and solution setting.

Transient solution and analysis of the results. Set the simulation time to $0.1 \mathrm{~s}$, and connect the external circuit to the transformer model according conditions which are set. Finite element analysis is carried out on the model. After the simulation, obtain series of simulation analysis data and analyze the rationality of the design scheme. If you are not satisfied with the results of simulation,you can modify the design and analyze the simulation again, until the desired design effect which we are excepted. Figure 6 to figure 8 is a balanced load simulation results (50 $\Omega$ resistance) [5-6]. 


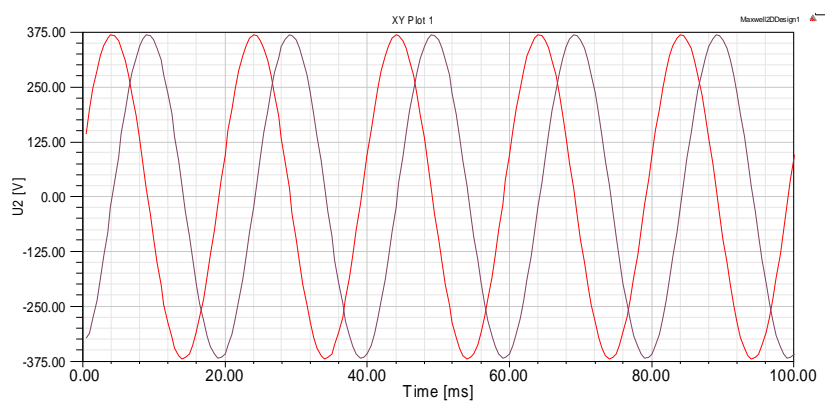

(a)

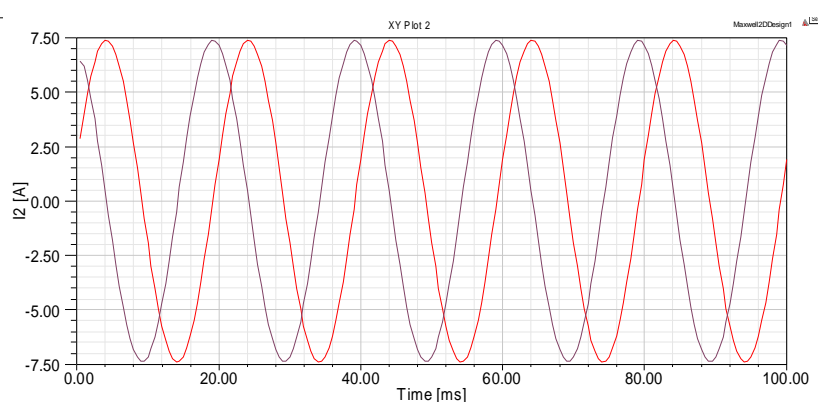

(b)

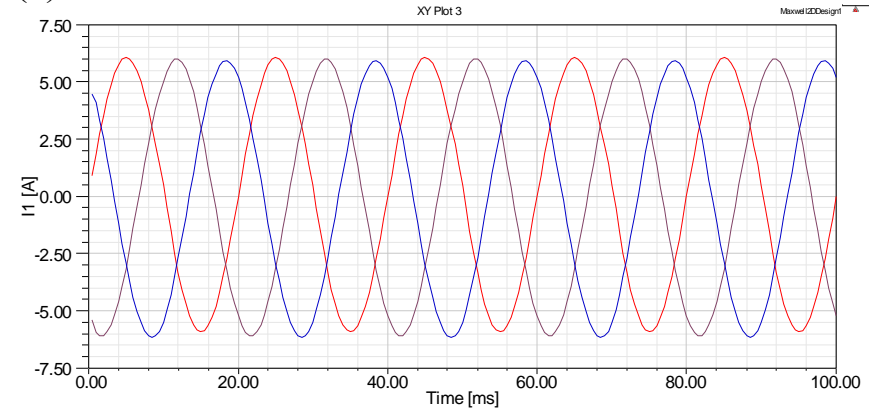

(c)

Fig. 6 Waves under balanced loads: (a) Waves of load voltage(b) Waves of load current (c) Waves of the primary phase current

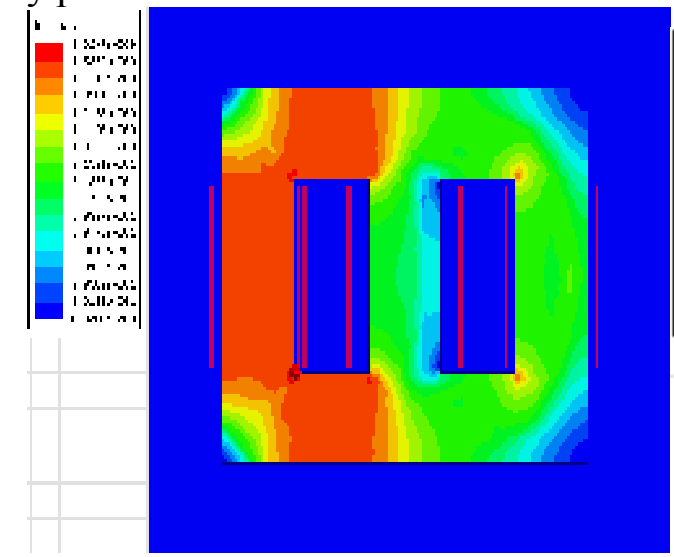

(a)

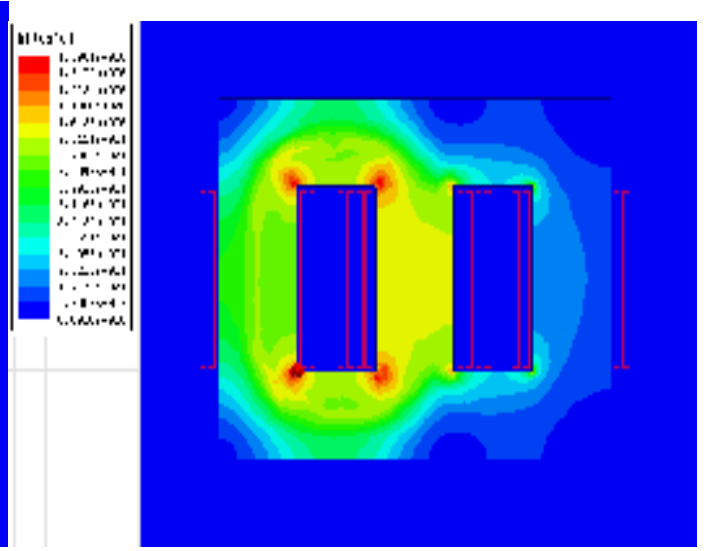

(b)

Fig. 7 Flux density cloud distribution in different time

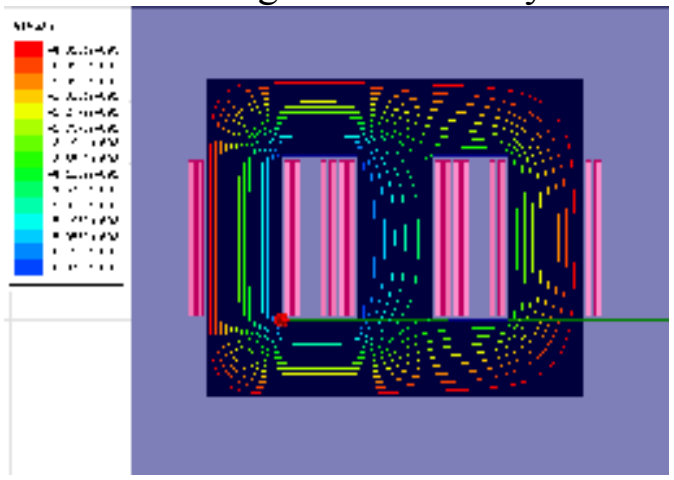

(c)

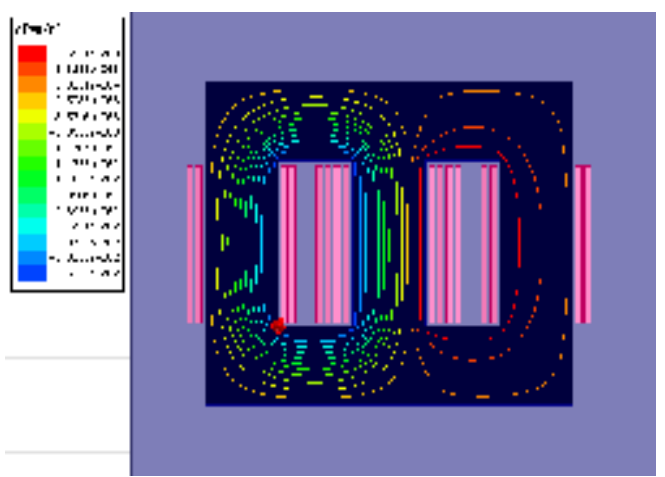

(d)

Fig. 8 Flux density distribution in different time when (a) $t=0.3 \mathrm{~s}(\mathrm{~b}) \mathrm{t}=0.45 \mathrm{~s}$ (c) $\mathrm{t}=0.3 \mathrm{~s}(\mathrm{~d}) \mathrm{t}=0.45 \mathrm{~s}$

Analysis of result . From the results, two phase load voltage, which are equal and the difference of phase is $90^{\circ}$, above that comply with the design requirements. At the same time under the condition of balanced load, the primary maintains symmetry state, which not only zero sequence current, but also do not contain negative sequence current, which realizes the basic function of balance transformer and the requirements of impedance matching. Through the flux density cloud distribution, we can intuitive 
observation internal dynamic parametersof transformer in different time, and verify the design of parameters.

\section{Conclusions}

(1)The results of Ansoft simulation of IMBF is consistent with the theoretical value, which shows that this simulation method is correct.

(2)Though Ansoft simulation, dynamic changes of the parameters be observed, which provides intuitive data for transformer optimization design.

(3) Using Ansoft, we not only can analyze the transient field, but also can analyze the static magnetic field, electrostatic field, eddy field and temperature field. So in practice, it can be used to make further research on the characteristics of the transformer.

\section{References}

[1] Q.Y. Liu, X.R. LI, C. Xiao, Research of impact of traction load negative sequence current on grid operating status, J. Advanced Technology of Electrical Engineering and Energy.4(2013)7-10.

[2] Y.L. Zhang, L.G. Chou. Electric analysis characteristic simulation of impendence-matching balance transformer, J. Electrical Engineering. 9(2014)36-39.

[3] R . Shipley, D . Coleman, C . Watts, Transformer circuits for digital studies, J. AIEE Trans., Pt. III. 81(1963)1028-1031.

[4] L. Zhou, X. Zhou, The analysis and research of phase shift rectifier transformer based on Ansoft, J. Journal of Technology University. 23(2009)66-69.

[5] Z.W. Xu, L.F. Luo , Z.W. Zhang, Three-to-four phase balance transformer with asymmetrical connection, J. Electric Power Automation Equipment.6(2012)14-18.

[6] Z.W. Xu, L.F. Luo , Z.W. Zhang, A novel asymmetrical connection three-phase to two-phase balance transformer, J. Proceedings of the CSEE. 32(2012) 148-154. 